\title{
Stability of the soliton states in a nonlinear fiber coupler
}

\author{
J. M. Soto-Crespo \\ Instituto de Óptica, Consejo Superior de Investigaciones Científicas, Serrano 121, 28006 Madrid, Spain \\ Nail Akhmediev \\ Optical Sciences Centre, Institute of Advanced Studies, The Australian National University, \\ Canberra, Australian Capital Territory 2601, Australia
}

(Received 17 May 1993)

\begin{abstract}
A detailed study of the stability of the soliton states for a nonlinear fiber coupler is presented. It is shown that the stability of the symmetric soliton states is delimited by the point of bifurcation: symmetric states are stable starting from zero energy up to the point of bifurcation, and unstable beyond the point of bifurcation. Asymmetric $A$-type states are stable at points with positive slope in the energy dispersion curve, and unstable otherwise. $B$-type asymmetric states are always unstable. Antisymmetric soliton states have regions of unstable as well as stable behavior. Moreover, the perturbation growth rate for the antisymmetric states can be complex in a certain range of spatial frequencies. A close analogy between the stability results for the soliton states in a nonlinear coupler and those for guided waves in planar nonlinear structures is established.
\end{abstract}

PACS number(s): 42.81.-i, 42.65.Re

\section{INTRODUCTION}

Soliton propagation in fiber waveguides supporting two coupled modes has been studied theoretically by several authors in the past few years. The nonlinear directional coupler has certainly been the most frequently studied such device since it was proposed [1,2]. Switching properties of this device, using very short pulses, have been studied extensively both theoretically [3-11] and experimentally [12-15]. It has been shown experimentally [15] that input pulses as short as $100 \mathrm{fsec}$ can be used to obtain complete switching. In principle, this is not the fastest switching time which can be achieved with directional couplers.

From a theoretical point of view, an important issue is the stationary pulses (soliton states) which can propagate in the coupler and their stability. The stability of the soliton states determines the device properties for long-term propagation and the behavior of pulses in the coupler. Symmetry-breaking instabilities in two-mode fiber devices taking into account (and also neglecting) cross-phase modulation have been studied by Wright, Stegeman, and Wabnitz [16]. It was shown in [16] that different types of behavior of the soliton pulses can be expected in the twomode devices depending on the perturbation of the initially stationary pulses.

Recently, Akhmediev and Ankiewicz [17] discovered different (asymmetric) families of soliton states in nonlinear fiber couplers. These soliton states are pairs of unequal pulses which can propagate in a directional coupler without changing their shapes. Moreover, the energydispersion diagram was constructed in [17] for all soliton states in terms of a unique parameter: their spatial frequency. This diagram shows that new states split off from symmetric and antisymmetric states at bifurcation points. Asymmetric states can play a decisive role in switching phenomena in nonlinear couplers.
In this paper we study numerically the stability of all soliton states in a nonlinear fiber coupler including asymmetric soliton states. We found the growth rates of all unstable branches and presented them in terms of the same soliton parameter. This allows us to establish a criterion of stability for the lowest branches of the soliton states. We find, for example, that the reason for symmetry-breaking instability [16] is the appearance of asymmetric soliton states at the point of bifurcation. Particularly, we find that symmetric soliton states are stable for energies below that corresponding to the bifurcation point $M$ [17], and are unstable otherwise. Asymmetric $\boldsymbol{A}$-type soliton states are stable at points with positive slope in the energy-dispersion curve, and unstable otherwise. Antisymmetric soliton states have regions of unstable as well as stable behavior. Moreover, the perturbation growth rate for the antisymmetric states can be complex in a certain range of spatial frequencies. The $B$ type asymmetric states are found to be always unstable.

The remainder of this paper is organized as follows: Section II describes the nonlinear coupler equations and their stationary solutions, together with the bases of the perturbative analysis. Section II is devoted to the study of the stability of the symmetric and antisymmetric states. $A$ - and $B$-type states are considered in Sec. IV. The analogies with the planar case are discussed in Sec. V. Finally, Sec. VI contains our conclusions.

\section{SOLITON STATES OF A NONLINEAR COUPLER}

Pulse propagation in a dual-core fiber coupler, including the effects of dispersion to second-order and selfphase modulation, can be described in terms of two linearly coupled nonlinear Schrödinger equations. In a reference frame traveling along the coupler with the light group velocity, this set of equations has the form [4] 


$$
\begin{aligned}
& i U_{\xi}+\frac{1}{2} U_{\tau \tau}+|U|^{2} U+K V=0, \\
& i V_{\xi}+\frac{1}{2} V_{\tau \tau}+|V|^{2} V+K U=0,
\end{aligned}
$$

where $U(\xi, \tau)$ and $V(\xi, \tau)$ are the electrical-field envelopes, $K$ is the normalized coupling coefficient between the two cores, $\xi$ is the normalized longitudinal coordinate, $\tau$ is the normalized retarded time, and the equations are written assuming anomalous group-velocity dispersion (GVD).

Separating explicitly the fast oscillatory part $e^{i q \xi}$ from the envelope functions, we can represent the solutions of Eq. (1) in the form

$$
\begin{aligned}
& U(\xi, \tau)=u^{\prime}(\xi, \tau) e^{i q \xi}, \\
& V(\xi, \tau)=v^{\prime}(\xi, \tau) e^{i q \xi},
\end{aligned}
$$

where $q$ is the signal wave-number shift and $u^{\prime}(\xi, \tau)$ and $v^{\prime}(\xi, \tau)$ are the new envelope functions. These functions are supposed to be free of fast oscillations in $\xi$. The value $q$ can be considered as the parameter of a soliton-state family of solutions. It is analogous to the propagation constant in the theory of nonlinear guided waves [18]. The remaining parameters of the soliton states depend on q. By substituting Eqs. (2) into Eqs. (1), one obtains

$$
\begin{aligned}
& i u_{\xi}^{\prime}+\frac{1}{2} u_{\tau \tau}^{\prime}-q u^{\prime}+\left|u^{\prime}\right|^{2} u^{\prime}+K v^{\prime}=0, \\
& i v_{\xi}^{\prime}+\frac{1}{2} v_{\tau \tau}^{\prime}-q v^{\prime}+\left|v^{\prime}\right|^{2} v^{\prime}+K u^{\prime}=0,
\end{aligned}
$$

which has two parameters $q$ and $K$. Using the following rescaling:

$$
u^{\prime}=u \sqrt{K}, \quad v^{\prime}=v \sqrt{K}, \quad t=\tau \sqrt{K}, \quad z=K \xi,
$$

Eqs. (3) become

$$
\begin{aligned}
& i u_{z}+\frac{1}{2} u_{t t}-\frac{q}{K} u+|u|^{2} u+v=0, \\
& i v_{z}+\frac{1}{2} v_{t t}-\frac{q}{K} v+|v|^{2} v+u=0,
\end{aligned}
$$

which have only one parameter, viz., $q / K$.

Equations (1) and (2) have the following invariant:

$$
\begin{aligned}
Q=\int_{-\infty}^{\infty}\left(|U|^{2}+|V|^{2}\right) d \tau & =\int_{-\infty}^{\infty}\left(\left|u^{\prime}\right|^{2}+\left|v^{\prime}\right|^{2}\right) d \tau \\
& =\sqrt{K} \int_{-\infty}^{\infty}\left(|u|^{2}+|v|^{2}\right) d t
\end{aligned}
$$

which corresponds to the total energy carried by a signal inside the fiber coupler. Equations (5) have stationary (independent of $z$ ) solutions: $u_{0}(t), v_{0}(t)$. These solutions can be found by solving Eq. (5), excluding the terms involving the derivatives with respect to $z$ :

$$
\begin{aligned}
& \frac{1}{2} u_{t t}-\frac{q}{K} u+|u|^{2} u+v=0, \\
& \frac{1}{2} v_{t t}-\frac{q}{K} v+|v|^{2} v+u=0 .
\end{aligned}
$$

Particularly, the set of Eqs. (7) has symmetric

$$
u_{0}=v_{0}=\frac{\sqrt{2(q / K-1)}}{\cosh [\sqrt{2(q / k-1)} t]}
$$

and antisymmetric

$$
u_{0}=-v_{0}=\frac{\sqrt{2(q / K+1)}}{\cosh [\sqrt{2(q / K+1)} t]}
$$

solutions.

In addition to symmetric and antisymmetric states, the set of Eqs. (7) also has asymmetric solutions with more complicated relations between the field components $u_{0}$ and $v_{0}$. There are no analytical expressions for them, but they can be found easily be numerical methods (see Ref. [17]). There are two different families of asymmetric solutions which bifurcate from the symmetric and antisymmetric states at certain values of $q / K$. They have been labeled in Ref. [17] as $A$ - and $B$-type asymmetric states. Figure 1(a), taken from Ref. [17], shows the dispersion curves on the $(Q, q)$ plane for symmetric, antisymmetric, and asymmetric soliton states. It is seen from Fig. 1(a) that the $A$ - and $B$-type asymmetric states split off from the symmetric and antisymmetric states at the bifurcation points $M$ and $N$, respectively.
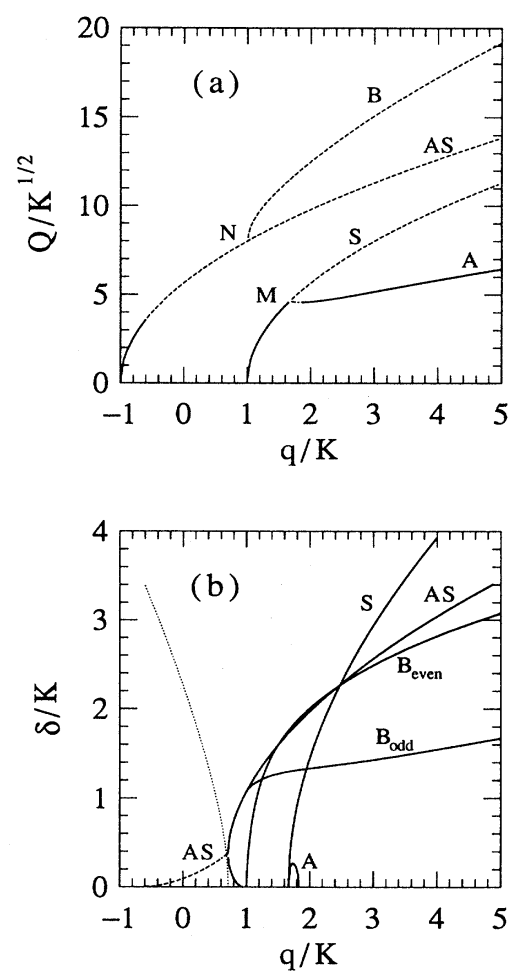

FIG. 1. (a) Total energy carried by the soliton states vs the normalized soliton parameter $q$. The solid lines correspond to stable branches, the dashed lines to unstable branches. Note that the curves for the symmetric $(S)$, antisymmetric $(A S)$, and $A$-type asymmetric ( $A$ ) soliton states have stable as well as unstable branches. $B$-type asymmetric and antisymmetric soliton states are represented by the curves $B$ and $A S$, respectively. $M$ and $N$ denote the bifurcation points. (b) Growth rates of unstable soliton states. The solid lines correspond to the purely real growth rates, the dashed line corresponds to the real part of the complex growth rate, and the dotted line corresponds to its imaginary part. The symbols above the curves in (b) correspond to those in (a). The $B$-type asymmetric soliton states have even and odd perturbation growth rates. 
Let the solutions of Eqs. (7), for some particular $q$, be denoted by $u_{0}(t)$ and $v_{0}(t)$. These could be either symmetric, antisymmetric, or asymmetric soliton states. We can study their stability by adding some small perturbations to them:

$$
\begin{aligned}
& u(z, t)=u_{0}(t)+\mu f(z, t), \\
& v(z, t)=v_{0}(t)+\mu h(z, t),
\end{aligned}
$$

where $f(z, t)$ and $h(z, t)$ are perturbation functions and $\mu$ is a small parameter. Substituting Eqs. (10) into Eqs. (5) and linearizing in the small parameter $\mu$, we obtain

$$
\begin{aligned}
& i f_{z}+\frac{1}{2} f_{t t}-(q / K) f+\left|u_{0}\right|^{2}\left(2 f+f^{*}\right)+h=0, \\
& i h_{z}+\frac{1}{2} h_{t t}-(q / K) h+\left|v_{0}\right|^{2}\left(2 h+h^{*}\right)+f=0 .
\end{aligned}
$$

This set of equations has many possible types of solution. We want to find those solutions which display exponential growth in the $z$ direction, and which are therefore unstable.

\section{STABILITY OF THE SYMMETRIC AND ANTISYMMETRIC SOLITON STATES}

For symmetric and antisymmetric states, $\left|u_{0}\right|=\left|v_{0}\right|$, and we have

$$
\begin{aligned}
& i f_{z}+\frac{1}{2} f_{t t}-(q / K) f+\left|u_{0}\right|^{2}\left(2 f+f^{*}\right)+h=0, \\
& i h_{z}+\frac{1}{2} h_{t t}-(q / K) h+\left|u_{0}\right|^{2}\left(2 h+h^{*}\right)+f=0 .
\end{aligned}
$$

From Eqs. (12) we have $h=+f$ or $h=-f$. Hence, four different combinations are possible. Let $f=h$ for symmetric states or $f=-h$ for antisymmetric states. Then the set (12) can be reduced to one equation. These two cases are equivalent to the problem of the stability of a soliton in a single fiber, which is known to be stable. Consequently, in this section we shall study the remaining two cases: $f=-h$ for the symmetric states and $f=h$ for the antisymmetric states.

In these two cases Eqs. (12) are reduced to a single equation,

$$
\begin{aligned}
i f_{z}+\frac{1}{2} f_{t t} & -(q / K \pm 1) f \\
+ & \frac{2(q / K \mp 1)}{\cosh ^{2}[\sqrt{2(q / K \mp 1)} t]}\left(2 f+f^{*}\right)=0,
\end{aligned}
$$

where the upper signs apply to the symmetric state and the lower signs apply to the antisymmetric state. We can simplify Eq. (13) by changing variables $t^{\prime}=t \sqrt{2(q / K \mp 1)}$ and $z^{\prime}=z(q / K \mp 1)$ :

$$
i f_{z^{\prime}}+f_{t^{\prime} t^{\prime}}-\eta_{ \pm} f+\frac{2}{\cosh ^{2} t^{\prime}}\left(2 f+f^{*}\right)=0
$$

where

$$
\eta_{ \pm}=\frac{(q / K \pm 1)}{(q / K \mp 1)}=\frac{(q \pm K)}{(q \mp K)} .
$$

We separate out the exponential dependence on $z^{\prime}$ and write the function $f\left(z^{\prime} t^{\prime}\right)$ in the form

$$
f\left(z^{\prime}, t^{\prime}\right)=\frac{1}{2}\left[(a+b) \exp \left(\gamma z^{\prime}\right)+\left(a^{*}-b^{*}\right) \exp \left(\gamma^{*} z^{\prime}\right)\right],
$$

where $\gamma$ is the growth rate of the perturbation, the asterisks denote complex conjugate, and the functions $a$ and $b$ depend only on $t^{\prime}$. Because of the double rescaling of variables, the growth rate $\gamma$ does not coincide with the growth rate $\delta^{\prime}$, which would be found by solving Eqs. (5), or $\delta$, which would be found solving directly Eqs. (3). Corresponding exponentials are $\exp \left(\delta^{\prime} z\right)$ in the case of Eqs. (5) and $\exp (\delta \xi)$ in the case of Eqs. (3). These growth rates are related by

$$
\delta=\delta^{\prime} K, \quad \delta=\gamma(q \mp K), \quad \delta^{\prime}=\gamma \frac{(q \mp K)}{K} .
$$

Substituting Eq. (15) in Eq. (14), we find that these functions must satisfy the following set of equations:

$$
\begin{aligned}
& a_{t^{\prime} t^{\prime}}-\eta_{ \pm} a+\frac{6}{\cosh ^{2} t^{\prime}} a=-i \gamma b, \\
& b_{t^{\prime} t^{\prime}}-\eta_{ \pm} b+\frac{2}{\cosh ^{2} t^{\prime}} b=-i \gamma a .
\end{aligned}
$$

Equations (17) can be solved analytically only for $\eta_{ \pm}=1$. In our case the value of $\eta_{ \pm}$varies in the range $[1, \infty]$ for symmetric states and in the range $[-1, \infty]$ for the antisymmetric states. The general solution cannot be found analytically and we have to solve Eqs. (17) numerically. We used the linearization method described in [19]. Let us first make some estimates at the limiting points. It is possible to find the solutions of Eqs. (17) when the growth rate is zero (i.e., $\gamma=0$ ). For the symmetric states the lowest (and unique) eigenfunction is an even function of $t$. Equations (17) have one approximate solution at $\eta_{+} \rightarrow 1$ $(q \rightarrow \infty)$ :

$$
\begin{aligned}
& a \approx\left(\frac{2 K}{q-K}\right)^{1 / 2} \frac{\cosh t^{\prime}-t^{\prime} \sinh t^{\prime}}{\cosh ^{2} t^{\prime}}, \\
& b \approx \frac{i}{\cosh t^{\prime}}, \\
& \gamma \approx\left(\frac{2 K}{q-K}\right)^{1 / 2},
\end{aligned}
$$

where $[2 K /(q-K)]^{1 / 2}$ is a small parameter.

Another point where $\gamma=0$ occurs at $\eta_{+}=4\left(q / K=\frac{5}{3}\right)$. This is exactly the point of bifurcation found in [17]. The functions $a$ and $b$ at this point are

$$
a=\frac{1}{\cosh ^{2} t^{\prime}}, \quad b=0 \text {. }
$$

Hence, $q=\frac{5}{3} K$ and $q \rightarrow \infty$ are the limiting values of instability for symmetric soliton states. The growth rates and perturbation functions between these two values can be found numerically. Our calculations show that the growth rate is real in this interval. The rescaled curve for the growth rate $\delta$ versus $q / K$ is shown in Fig. 1(b) by the solid line labeled $S$. In the interval $K<q<5 K / 3$, the set of Eqs. (17) has only imaginary eigenvalues $\gamma$. As a result, the symmetric soliton states are stable up to the point of bifurcation and unstable beyond the point of bifurcation, i.e., they are unstable at $q$ values where asymmetric $A$-type soliton states exist. 
For the antisymmetric soliton states, there are even and odd perturbation functions. The odd perturbation function has the largest growth rate. The value $\eta_{-}=1$ $(q \rightarrow \infty)$ is one of the limiting values where $\gamma=0$ for the odd eigenfunction. The eigenfunctions of the perturbation $a$ and $b$ at this point are

$$
a=\frac{\sinh t}{\cosh ^{2} t}, \quad b=0
$$

The other value of $q$ where $\gamma \rightarrow 0$ is $q=K$. The behavior of the eigenvalue corresponding to the odd perturbation function is more complicated close to this point. The growth rate $\delta$ increases to the left of this point. In the region $K / 1.4<q<K$, two different eigenvalues associated to odd eigenfunctions exist. Numerical simulations show that the antisymmetric state has real eigenvalues $\delta$ in the whole interval $K / 1.4<q<\infty$. In addition to them, there are complex eigenvalues $(\delta+i \Omega)$ in the interval $-0.6 K<q<K / 1.4$. This set of eigenvalue curves, labeled $A S$, is shown in Fig. 1(b). The dashed and the dotted lines stand for the real and the imaginary parts, respectively, of the eigenvalue in the region where it is not purely real: $-0.6 K<q<K / 1.4$. The eigenfunctions corresponding to these curves are odd functions of $t$ for all $q$. As a result, the antisymmetric states are unstable in the range $-0.6 K<q<\infty$. We did not find numerically real eigenvalues in the range $-K<q<-0.6 K$. Hence, the antisymmetric state at small energies is stable.

There are even eigenfunctions corresponding to antisymmetric states as well. The range of $q$ values where these eigenfunctions exist is located inside the range where odd perturbation functions exist. Their eigenvalues are lower than those for odd functions. Hence, their influence on the field evolution in nonlinear couplers will be less pronounced. We leave the study of these states for future publications. Examples of perturbation functions for the symmetric and antisymmetric soliton states are given in Figs. 2 and 3.

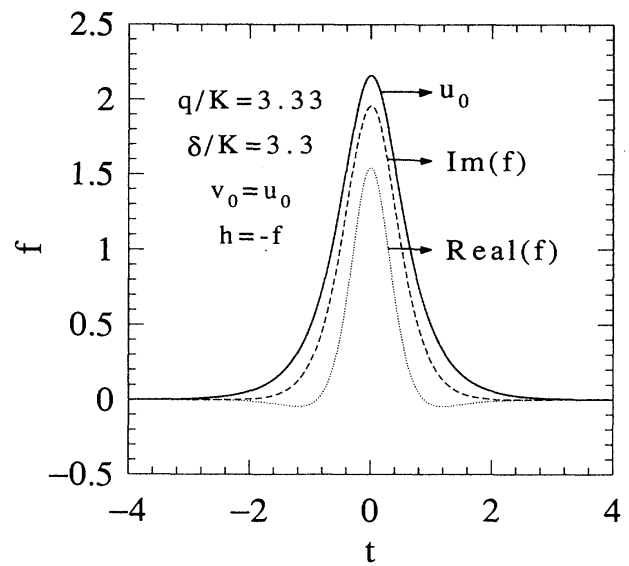

FIG. 2. Real (dotted line) and imaginary (dashed line) parts of the perturbation $[f(\tau)=-h(\tau)]$ of the symmetric state at the point $q / K=3.33$. The shape of the stationary symmetric state $u_{0}=v_{0}$ is shown by a solid line.

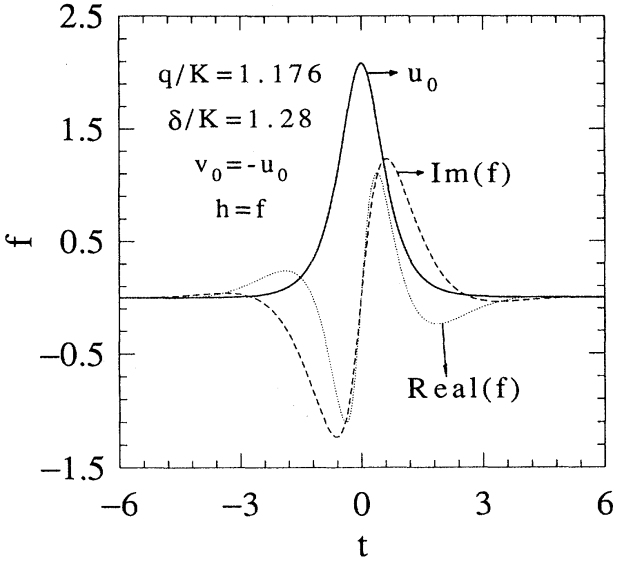

FIG. 3. Real (dotted line) and imaginary (dashed line) parts of perturbation $[f(\tau)=h(\tau)]$ for the antisymmetric state at the point $q / K=1.176$. The shape of the stationary antisymmetric state $u_{0}=-v_{0}$ is shown by a solid line for comparison.

\section{STABILITY OF ASYMMETRIC $A$ - AND $B$-TYPE SOLITON STATES}

In the case of asymmetric soliton states, $\left|u_{0}\right| \neq\left|v_{0}\right|$. Therefore, Eqs. (11) cannot be reduced to a single equation, and we have to solve them numerically in the entire range of $q$ where a certain type of stationary solution exists. We used the linearization method described in [19] modified for the case of a coupled set of evolution equations. In this way we can find the perturbation function possessing a given symmetry with the largest growth rate. The results for $A$ - and $B$-type asymmetric soliton states are shown in Fig. 1(b) (the curves are denoted as $A, B_{\text {even }}$, and $\left.B_{\text {odd }}\right)$. In the case of asymmetric $A$-type soliton states, the eigenvalue $\delta$ is purely real in a small region in the interval $5 K / 3<q \lesssim 1.85 K$ (curve $A$ ). The corresponding eigenfunctions $(f, h)$ are even functions of $t$. Hence, $A$-type soliton states are unstable immediately beyond the point of bifurcation (point $M$ ). The value $q=q_{\min } \approx 1.85 K$ corresponds to the point where the energy dispersion curve $Q(q)$ [see Fig. 1(a)] for the $A$-type states has a minimum. This means that the asymmetric $A$-type soliton states are unstable at the values of $q$ where $d Q / d q$ is negative. The eigenvalue $\delta$ is purely imaginary at $q>q_{\min } \approx 1.85 K$, where $d Q / d q$ is positive. Hence, the $A$-type states are stable in this part of the dispersion curve.

In the case of the asymmetric $B$-type states, there are two different (even and odd) bound perturbation functions. The growth rates $\delta$ for each of them are real in the entire region where $B$-type soliton states exist. Two $\delta$ curves corresponding to these two perturbation functions are shown in Fig. 1(b) (curves $B_{\text {even }}$ and $B_{\text {odd }}$ ). The curve for $\delta / K$ vs $q / K$ corresponding to the even perturbation function goes to zero at the point of bifurcation $q=K$ (point $N$ ). The odd perturbation function at the point of bifurcation (point $N$ ) has the same growth rate as that for odd perturbations of antisymmetric soliton states. This shows that the unstable behavior of the $B$-type states is 
the continuation of the unstable behavior of the antisymmetric states up to point $N$.

As both eigenvalues are real in the whole region where $B$-type states exist, these states are all unstable. The two eigenvalues for even and odd perturbation functions are of the same order of magnitude. Moreover, there is a point $q=1.25 \mathrm{~K}$, where their values are exactly equal. This means that the evolution of unstable $B$-type states can be quite complicated in general.

\section{STABILITY CRITERION AND ANALOGY WITH GUIDED WAVES IN PLANAR STRUCTURES}

The above results concerning the stability of the different types of soliton states are summarized in Fig. 1(a). The continuous curves show the stable branches and the dashed curves, the unstable branches. There is not a general stability criterion for all types of soliton states. Nevertheless, it is possible to reach certain conclusions.

For the lower-order (symmetric and $A$-type asymmetric) soliton states, the stability criterion can be expressed as follows: If two different soliton states exist at a given $q$, the state with higher energy is unstable. The lowest (in energy) soliton state is stable at $d Q / d q \geq 0$, and unstable otherwise. This criterion means that $A$-type asymmetric states, which have minimum energy at a certain $q=q_{\text {min }}$, are stable at $q>q_{\text {min }}$ and unstable at $q_{M}<q<q_{\min }$, where $q_{M}$ is the value of $q$ at point $M$. The symmetric soliton states are stable up to the point of bifurcation (point $M$ ) and unstable beyond it, as they then have higher energies than the corresponding $A$-type states. Thus, in the small range $\frac{5}{3}<q / K<1.85$, there are no stable states at all.

There is no such simple criterion for the stability of higher-order soliton states. Each case has to be considered separately. $B$-type asymmetric soliton states are unstable in the whole region where they exist. Antisymmetric states have regions of unstable as well as stable behavior. Furthermore, we found that the eigenperturbations of the antisymmetric states can possess complex eigenvalues.

There is a close analogy between the soliton states in a nonlinear directional coupler and the nonlinear guided waves in a symmetric planar waveguide structure with a linear layer [18]. The bifurcation phenomena and stability properties are very similar if we consider $q$ as an analog of the propagation constant in a planar waveguide structure, and the total energy $Q$ in the directional coupler as an analog of the energy flow (or power flow) in the planar waveguides [18]. Hence, we can deduce some general properties of the solutions from one case and apply them to the other, because physically these two problems are quite similar. Basically, in each case, nonlinear waves in two media (or cores) interact linearly through the evanescent optical fields. However, we have to bear in mind the three main differences between these problems. First, the solitons in a directional coupler are temporal solitons rather than spatial solitons. Second, the linear interaction is distributed along the pulses in the fiber coupler, but is concentrated on the interfaces in planar waveguides. Third, pulse propagation in a coupler has to be described by two complex functions, in contrast to the waveguide, where we deal with a single complex function. As a result, more symmetry relations are involved in our problem than in the case of the planar waveguide.

Let us notice some common features between our stability results for the directional coupler and those for planar waveguides. We can consider the $Q$ versus $q$ diagram of the present paper as the analog to the $I$ versus $n$ diagram for planar waveguides [20]. In this case the stability criterion for the symmetric and $A$-type asymmetric soliton states found in the present investigation is practically the same as that for planar waveguides $[21,20,22]$. Additionally, in both problems the perturbation eigenvalues can be complex for antisymmetric waves [23].

We also notice some differences in the stability results. The dispersion curves for each particular type of soliton state cannot be $N$ shaped in the directional coupler, in contrast to the planar waveguide [18]. Another difference is that the higher-order nonlinear modes have different stability properties. For example, antisymmetric states in planar waveguides can be stable at large values of the propagation constant [23]. Antisymmetric states in the coupler are unstable at high $q$. In both cases the stability criterion for higher-order stationary solutions (soliton states) cannot be expressed in simple terms [23].

\section{CONCLUSIONS}

In conclusion, we have studied the stability of the soliton states in nonlinear fiber couplers. A stability criterion has been established for lower-order soliton states (symmetric and asymmetric $A$-type states). Asymmetric $B$-type states are found to be always unstable. Perturbation eigenmodes associated with the antisymmetric states are shown to have complex eigenvalues in some range of soliton parameter. Similarities and differences with guided waves in a planar geometry are demonstrated.

\section{ACKNOWLEDGMENTS}

One of the authors (N.A.) thanks Dr. A. Ankiewicz for useful discussions. The work of J.M.S.-C. was supported by the CICyT under Contract No. TIC91-0361. The work of N.A. is supported by the Australian Photonics Cooperative Research Centre (APCRC).
[1] A. A. Maier, Kvant. Elektron. (Moscow) 9, 2296 (1982) [Quantum Electron. 12, 1490 (1982)].

[2] S. M. Jensen, IEEE J. Quantum Electron. QE-18, 1580 (1982).
[3] S. Trillo, S. Wabnitz, E. M. Wright, and G. I. Stegeman, Opt. Lett. 13, 672 (1988).

[4] G. D. Peng and A. Ankiewicz, Int. J. Nonlinear Opt. Phys. 1, 135 (1992). 
[5] F. Kh. Abdullaev, R. M. Abrarov, and S. A. Darmanyan, Opt. Lett. 14, 131 (1989).

[6] A. Ankiewicz and G. D. Peng, Int. J. Optoelectron. 6, 15 (1991).

[7] F. Kh. Abdullaev and A. A. Abdumalikov, Pis'ma Zh. Tekh. Fiz. 14, 1041 (1988) [Sov. Tech. Phys. Lett. 14, 458 (1988)].

[8] J. M. Soto-Crespo and E. M. Wright, J. Appl. Phys. 70, 1365 (1989).

[9] P. L. Chu, G. S. Peng, and B. A. Malomed, Opt. Lett. 18, 328 (1993).

[10] Y. S. Kivshar, Opt. Lett. 18, 7 (1993).

[11] M. Romangnoli, S. Trillo, and S. Wabnitz, Opt. Quantum Electron. 24, S1237 (1992).

[12] D. D. Gusovskii, E. M. Dianov, A. A. Maier, V. B. Neustruev, V. V. Osiko, A. M. Prokhorov, K. Yu. Sitarskii, and I. A. Scherbakov, Kvant. Electron. (Moscow) 14, 1144 (1987) [Sov. J. Quantum Electron. 17, 724 (1987)].

[13] A. A. Maier, Yu. N. Serdyuchenko, K. Yu. Sitarskii, M. Ya. Shchelev, and I. A. Scherbakov, Kvant. Electron. (Moscow) 14, 1157 (1987) [Quantum Electron. 17, 735 (1987)].
[14] S. F. Friberg, Y. Silberberg, M. K. Oliver, M. J. Andrejco, M. A. Saifi, and P. W. Smith, Appl. Phys. Lett. 51, 1135 (1987).

[15] S. F. Friberg, A. M. Weiner, Y. Silberberg, B. G. Sfez, and P. S. Smith, Opt. Lett. 13, 904 (1988).

[16] E. M. Wright, G. I. Stegeman, and S. Wabnitz, Phys. Rev. A 40, 4455 (1989).

[17] N. N. Akhmediev and A. Ankiewicz, Phys. Rev. Lett. 70, 2395 (1993).

[18] N. N. Akhmediev, Zh. Teor. Eksp. Fiz. 83, 545 (1982) [Sov. Phys. JETP 56, 299 (1982)].

[19] N. N. Akhmediev, V. I. Korneev, and Yu. V. Kuz'menko, Zh. Eksp. Fiz. 88, 107 (1985) [Sov. Phys. JETP 72, 619 (1985)].

[20] N. N. Akhmediev and N. V. Ostrovskaja, Zh. Tekh. Fiz. 58, 2194 (1988) [Tech. Phys. 33, 1333 (1988)].

[21] C. K. R. T. Jones and J. Moloney, Phys. Lett. A 117, 175 (1986).

[22] D. J. Mitchell and A. W. Snyder, J. Opt. Soc. Am. B 10, 1572 (1993).

[23] N. N. Akhmediev, A. Ankiewicz, and H. T. Tran, J. Opt. Soc. Am. B 10, 230 (1993). 